\title{
HIV-1 entry inhibitors and virus emergence
}

To the editor-In their recent News and Views article ${ }^{1}$, Michael and Moore concluded that "given recent demonstrations of both viral resistance and persistent provirus in the face of the anti-retroviral drug regimes now in use, the careful clinical assessment of HIV-1 entry inhibitors is urgently needed." Although we agree wholeheartedly with this conclusion, we have data that challenge one of their assumptions regarding the emergence of alternative HIV-1 variants.

Michael and Moore state "the frequency with which X4 [CXCR4-tropic] viruses emerge in individuals who are heterozygous for the CCR5 $\Delta 32$ allele is no greater than in individuals with a wild-type allele $^{2}$, despite the reduced CCR5 expression associated with the CCR5 $\Delta 32$ heterozygous genotype." The study they cite examined newly infected patients ${ }^{2}$. This is important, as there are other data to suggest that the relatively slow disease progression seen in CCR5 $\Delta 32$ heterozygotes is limited to the first few years after infection ${ }^{3}$. Among the moderately advanced HIV-infected patients who entered the NIAID AIDS clinical trials group protocol 241, baseline syncytium-inducing (SI) viral phenotype was more common among the CCR5/ $\Delta$ ccr5 heterozygotes (7of 7, 100\%) than among the CCR5/CCR5 homozygotes (29 of $88,33 \% ; P<0.001$, Fisher's exact test). The SI phenotype indicates tropism for cells expressing CXCR4 or both CCR5 and CXCR4, rather than for cells expressing only CCR5. Other factors that might explain this observation did not differ at study entry by CCR5 genotype, including median CD4 cell counts, plasma HIV-1 RNA levels, infectious HIV1 titer in peripheral blood mononuclear cells, and history of prior anti-retroviral treatment ${ }^{4}$. These data are consistent with that possibility that reduced functional availability of CCR5 will increase selection for X4-tropic, or dual-tropic, viruses after several years of infection.

Given these data, we would like to further emphasize the need for a careful assessment of potential CCR5-active inhibitors as components of combination regimens. We also feel that an inhibitor active against CXCR4 would be a theoretically attractive agent to combine with a CCR5-active inhibitor, to attempt to limit broadening of co-receptor usage as a viral escape route.
RichaRd T. D'AQUILA ${ }^{1}$, MiCHAEL Hughes $^{2} \&$ Victoria A. JOHNSON ${ }^{3}$ ${ }^{1}$ Infectious Disease Division and AIDS Research Center, MGH

Charlestown, Masschusetts 02129, USA

${ }^{2}$ Harvard School of Public Health

Dept. of Biostatistics

655 Huntington Ave., 4th Floor

Boston, Massachusetts 02115, USA

${ }^{3}$ Div. of Infectious Diseases

Depts. of Medicine and Microbiology

University of Alabama at Birmingham School of Medicine

229 Tinsley Blvd

Birmingham, Alabama 35294-0006, USA

1. Michael NL, Moore JP. HIV-1 entry inhibitors: Evading the issue. Nature Med. 5, 740-742 (1999).

2. Michael, N.L. et al. The role of viral phenotype and CCR-5 gene defects in HIV-1 transmission and disease progression. Nature Med. 3, 338-340 (1997)

3. Eugen-Olsen I. et al. Heterozygosity for a deletion in the CKR-5 gene leads to prolonged AIDS-free survival and slower CD4 T-cell decline in a cohort of HIV-seropositive individuals. AIDS 11, 305-310 (1997).

4. D'Aquila RT, Sutton L, Savara A, Hughes MD \& Johnson VA, for the NIAID AIDS Clinical Trials Group protocol 241 Virology Team. CCR5/delta ccr5 heterozygosity: A selective pressure for the syncytium-inducing human immunodeficiency virus type 1 phenotype. J. Infect. Dis. 177,1549-53 (1998).

\section{Japan's tobacco tragedy}

To the editor-Your August editorial ${ }^{1}$ calls attention to one of the most dire threats to global health-smoking. According to the World Health Organization (WHO), four million people die each year from tobacco-related illness, and that figure is expected to rise to about ten million by the year 2030 (ref. 2).

In Japan in 1998, the number of deaths due to lung cancer $(50,867)$ exceeded stomach cancer deaths $(50,662)$, making lung cancer Japan's leading cause of death due to malignant tumors $^{3}$. Lung cancer now accounts for $17.8 \%$ of all cancer deaths - a tenfold increase since 1950. Although tobacco undoubtedly contributes to this increase, Japan has done little to respond to this growing problem, and as a result lung cancer and other tobacco-related diseases are on the rise.

The WHO estimated that in 1994, $59.0 \%$ of Japanese men and $14.8 \%$ of women smoked. Although the percentage of male smokers has dropped from a staggering $83.7 \%$ in 1966 , it is still far higher than that in many other developed countries (and almost equals that in China (61.0\% in men), a country that is now facing an immense problem with tobacco ${ }^{4}$. Although a smaller percentage of Japanese women smoke than in most developed countries, the rate is not falling. Even more worrying is a demographic switch that sees an increase in the proportion of younger women smokers ${ }^{5}$. Smoking among Japanese teenagers is also a serious concern. Although Japan prohibits smoking until the age of 20, this legislation is mostly ineffective, as tobacco is readily available to teenagers through vending machines and convenience stores.

In Japan, the number of excess deaths due to tobacco is estimated at around 100,000 per year ${ }^{5}$. Although this is more than 10 times the number of deaths due to road traffic accidents, resources and efforts allocated for tobacco control are minimal compared with those allocated for traffic safety. Smoking as a cause of death has been neglected in spite of the fact that it is our leading preventable cause of death. It is time for Japan to take effective action to stop tobacco from killing yet another generation.

KAZUO INOUE

Towa Clinic

Dept. of Community and Family Medicine Jichi Medical School

468 Showa, Towa, Hata

Kochi 786-0511 Japan

E-mail ikazu@ps.inforyoma.or.jp

1. Tobacco control-drawing breath. Nature Med. 5, 851 (1999).

2. Roemer, R. Legislative Action to Combat the World Tobacco Epidemic (World Health Organization, Geneva, 1993).

3. Ministry of Health and Welfare. The 1998 Vital Statistics of Japan (the Ministry, Tokyo, 1999).

4. Peto, R., Chen, Z.M. \& Boreham, J. Tobacco-the growing epidemic. Nature Med. 5, 15-17 (1999).

5. Ministry of Health and Welfare. Smoking and Health (White paper on tobacco) (Japan Health Promotion and Fitness Foundation, Tokyo, 1993). 